\title{
Quantitative Characterization of Precipitate Microstructures in Metallic Alloys Using Small-Angle Scattering
}

\begin{abstract}
ALEXIS DESCHAMPS and FRÉDÉRIC DE GEUSER
Quantitatively characterizing precipitate microstructures in metals by small-angle scattering poses specific challenges as compared to other areas of application of this technique. In terms of size and morphology evaluation, these include the presence of a significant size distribution, non-isotropic shapes, and interpretation complicated by a partial averaging due to a nonrandom texture. In terms of volume fraction evaluation, these include the imperfect knowledge of the chemical composition of very small objects. This paper, based on a presentation given at the "Neutron and X-Ray Studies of Advanced Materials V: Centennial" symposium of the 2012 TMS conference, reviews the strategies that can be applied in different characteristic cases to obtain a robust quantification of precipitate microstructures.
\end{abstract}

DOI: $10.1007 / \mathrm{s} 11661-012-1435-7$

(C) The Minerals, Metals \& Materials Society and ASM International 2012

\section{INTRODUCTION}

WHEN metallic alloys are probed by an X-ray or neutron beam, local fluctuations at the nanometer scale of the density of scattering factors (electrons for X-rays, atomic nuclei or magnetic moments for neutrons) result in small-angle scattering (SAS). Such fluctuations are most often related to fluctuations in chemical composition, themselves due to the presence of precipitates. Of course, other sources of scattering may exist such as nanopores or crystalline defects.

Small-angle scattering has been used almost since the discovery of precipitation strengthening in metals to characterize the presence of the precipitates. Progressively, largely following the pioneering work of Guinier, ${ }^{[1,2]}$ it has been used to quantify the precipitate microstructures, namely their size, size distribution, or volume fraction. A number of review articles have been written since then over the decades that follow the improvements of the analysis techniques and of the instruments. ${ }^{[3-5]}$

Nowadays, numerous models, embedded in available software, are available to extract structural data from a SAS spectrum. Because the SAS community is currently largely dominated by studies of organic compounds, a comparatively small number of available programs are really adapted to the case of precipitation in inorganic materials. A SAS measurement (like any diffraction measurement) is equivalent to a measurement of the square of the modulus of the Fourier transform of the density of the scattering entities present in the sample. Thus, the two main strategies for extracting structural

ALEXIS DESCHAMPS, Professor, is with INP Grenoble, Grenoble, France. Contact e-mail: alexis.deschamps@grenoble-inp.fr FRÉDÉRIC DE GEUSER, Research Scientist, is with SIMAP, CNRS, Grenoble, France.

Manuscript submitted April 18, 2012.

Article published online October 2, 2012 information from SAS spectra are the direct and reverse methods.

In the direct method, a certain distribution function of the precipitates is assumed, the scattering of which can be calculated and fitted to the experimental data as a function of the parameters of this function. For instance, a log-normal size distribution of precipitates is often assumed in the literature, ${ }^{[6-10]}$ the size and dispersion of which can be adjusted. Other distributions, or combinations of several distributions, can naturally be used as well. ${ }^{[1-15]}$ In the indirect method, an inverse Fourier transform of the data is made, resulting in a presumably assumption-free measurement of the precipitate size distribution (see e.g. ${ }^{[16,17]}$ ).

However simple in principle, and widespread in the literature, these techniques are still challenging when dealing with microstructures of "real" materials, namely materials with several length scales of particles, complicated particle shapes, high volume fractions, etc. Among the many difficulties that can arise one can cite the following:

(a) Lack of proper measurements at both ends of the scattering range: At small angles, the measurements are often perturbed by some parasitic scattering due to double Bragg scattering, scattering from large precipitates, e.g., at the grain boundaries, or structural defects (e.g., high dislocation densities). ${ }^{[18,19]}$ These contributions should be subtracted, whenever possible, but their intensity is difficult to establish with high precision. At large angles, the measurements may be of low precision due to poor measurement statistics, fluorescence of some elements of the microstructure at the measurement wavelength, and imperfections in the detector corrections.

(b) High volume fraction: In the case of high volume fraction of particles, the scattering from neighboring objects interferes and the scattering function deviates from the addition of the individual 
scattering functions from the different objects. ${ }^{[20,21]}$ The case of high volume fractions with monodisperse objects has been properly described by a number of models, however, a full description of the combined effects of high volume fraction and precipitate size distributions remains challenging.

In parallel, simple methods of precipitate size measurement exist, like the measurement of the "Guinier" radius, and are extensively used in the literature. ${ }^{[18,22-27]}$ However, for a proper use of these measurements, it is important to ascertain their validity and establish their limits.

The aim of the present paper is to review some strategies for measuring precipitate microstructures in different situations. In Section I-A, we will review the case of particles of spherical or near-spherical shape (like low aspect ratio ellipsoids or platelets). In Section I-B, we will review the case of platelet precipitates of high aspect ratio, when the scattering signal is reasonably isotropic due to an averaging over all grain orientations within the probed volume. In Section I-C, we will discuss the case of platelet particles, when the grain averaging of the scattering is sufficiently low so that the anisotropic signature of the platelets is still measurable. Finally, in Section I-D, we will discuss the complications that arise when a measurement of volume fraction is sought.

\section{A. Spherical or Near-Spherical Precipitates}

The scattering of a single sphere can be exactly calculated:

$$
I(q, R)=\left[3\left(\rho_{\mathrm{p}}-\rho_{\mathrm{m}}\right) V_{\mathrm{p}}\left(\frac{\sin (q R)-q R \cos (q R)}{(q R)^{3}}\right)\right]^{2}
$$

where $q=4 \pi \sin \theta / \lambda$ is the scattering vector, $R$ the radius of the particle, $\rho_{\mathrm{p}}$ and $\rho_{\mathrm{m}}$ are the density of scattering factors in the precipitate and matrix, respectively, and $V_{\mathrm{p}}$ is the particle volume. Note that for the case of an ellipsoid, the scattering function can be exactly calculated as well as that originating from a sphere of a radius depending on the orientation of the ellipsoid with respect to the beam. ${ }^{[8]}$ Thus, when an isotropic distribution of such particles is probed (namely particles the orientations of which are isotropically averaged over all possible orientations), the total scattering can be calculated by simple integration over the precipitate size distribution and the orientations in space.

When $q \rightarrow 0$, this equation can be approximated by the Guinier approximation:

$$
I(q, R) \cong\left(\left(\rho_{\mathrm{p}}-\rho_{\mathrm{m}}\right) V_{\mathrm{p}}\right)^{2} \exp \left(-\frac{q^{2} R_{\mathrm{g}}^{2}}{3}\right)
$$

where $R_{\mathrm{g}}$ is the gyration (or often called Guinier) radius of the particle, the value of which can therefore be easily measured by the low- $q$ limit of the slope of the so-called Guinier plot $\ln (I) v s q^{2}$. In the case of monodispersed spheres, the Guinier radius is related to the radius of the sphere by a proportionality factor $R_{\mathrm{g}}=\sqrt{3 / 5} R$.

However, metallurgical systems very seldom present monodisperse particle distributions. In the presence of a precipitate size distribution, the scattering behavior is modified in the complete $q$-range, including in the low- $q$ regime where the Guinier radius is measured. Actually, the size dispersion acts both on the extension of the range of the Guinier approximation (Gaussian approximation or straight line in the Guinier plot) and on the value of the measured Guinier radius. ${ }^{[28]} \mathrm{In}$ many practical cases, precipitate size distributions $f(R)$ can be adequately described by log-normal distributions:

$$
f(R)=\frac{1}{\sqrt{2 \pi} s R} \exp \left(-\frac{1}{2}\left(\frac{\ln \left(R_{m} / R\right)}{s}\right)^{2}\right)
$$

Interestingly, the maximum extension of the linear regime in the Guinier plot is observed when the dispersion parameter $s$ of the log-normal distribution is equal to 0.2 (approximately 20 pct relative standard deviation from the average size). ${ }^{[28]}$ Moreover, in this case, the measured Guinier radius is precisely equal to the average radius of the precipitate size distribution. Such a dispersion parameter is actually close to what is found in many practical cases, which explains that usually, the measurement of the Guinier radius is made well beyond the theoretic limit for a single sphere (up to $q \cdot \mathbf{R}_{g}=3$ instead of the limitation $q \cdot \mathbf{R}_{g} \ll 1$ for the monodisperse case). For this reason, in somewhat concentrated systems, it is still possible to measure the value of the Guinier radius beyond the intensity maximum resulting from particle interference because of this large extension. Also, this extension makes it possible to use an alternative way to measure the Guinier radius (sometimes called the Pseudo-Guinier radius) from the scattering vector where $I \cdot q^{2}$ goes through a maximum (identified in the Kratky plot $I \cdot q^{2}$ vs q). ${ }^{[28,29]}$

Thus practically, in systems containing a distribution of spheres of reasonable dispersion (with a dispersion parameter between 0.15 and 0.25 ) or non-spherical objects of a relatively small aspect ratio (for a rue of thumb let say not more than 2), the measurement of the Guinier radius, if made in a self-consistent way as presented in, ${ }^{[28]}$ provides a robust measurement of the average precipitate radius without the need of a proportionality factor.

As an example of this measurement procedure, Figure 1(a) shows the Guinier plots corresponding to SAXS measurements (on a laboratory set-up) of an Al$\mathrm{Mg}-\mathrm{Li}$ alloy subjected to two different aging conditions, where spherical $\mathrm{Al}_{3}(\mathrm{Mg}, \mathrm{Li}) \mathrm{Ll}_{2}$ precipitates form in large volume fraction (for more details regarding alloys, heat treatment, and measuring conditions refer to ${ }^{[30]}$ ). In relation to the size dispersion of the particles, and despite the interference peak due to the high precipitate volume fraction and small precipitate size, an extended straight line in the Guinier plot is observed. The size extracted from this slope is completely consistent with 


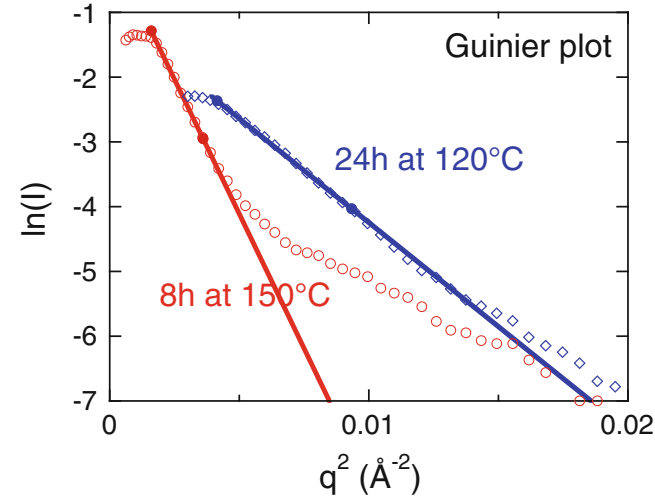

(a)

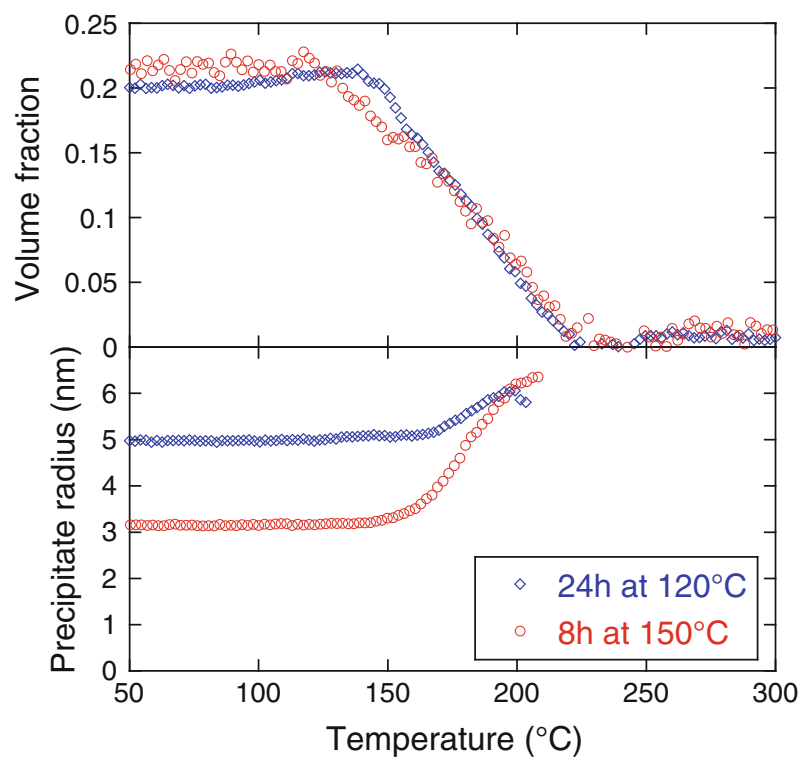

(b)

Fig. 1-(a) Guinier plots of the SAXS signal in an Al-Mg-Li alloy for two aging conditions; $(b)$ evolution of the precipitate radius and volume fraction during ramp heating experiments at $0.5 \mathrm{~K} \mathrm{~s}^{-1}$ on these two initial aging conditions.

that obtained by transmission electron microscopy (TEM) measurements. ${ }^{[30]}$ Using this procedure, large datasets can be easily processed, such as that obtained during in situ heat treatments under the X-ray beam. Figure 1(b) shows the evolution of the size and volume fraction of these precipitates when the two precipitate microstructures presented above are subjected to a heating ramp at a rate of $0.5 \mathrm{~K} \mathrm{~s}^{-1}$. The evolution of the precipitate microstructures can be successfully compared to a numerical model and can be understood in terms of the evolution of the precipitate size distribution when the temperature becomes closer to the solvus of the alloy. ${ }^{[30]}$

\section{B. Platelet Precipitates: Radial Averaging of the Intensity}

In many alloy systems, precipitates are present in the shape of platelets of a high aspect ratio. This is the case, for instance, in Aluminum alloys (GPI, GPII, and $\theta^{\prime}$ phases in the Al-Cu system ${ }^{[31]}$ or $T_{1}$ phase (nominally $\mathrm{Al}_{2} \mathrm{CuLi}$ ) in the $\mathrm{Al}-\mathrm{Cu}-\mathrm{Li}$ system $)^{[32]}$ or in $\mathrm{Mg}$ alloys (e.g., $\beta$ and $\beta_{1}$ phases in $\mathrm{Mg}-\mathrm{Y}-\mathrm{Nb}^{[33]}$ or GP zones in $\mathrm{Mg}-\mathrm{Ca}-\mathrm{Zn}){ }^{[34]}$ When the scattering of such platelets is relatively well averaged over all possible orientations, a radial averaging of the scattered intensity gives access to different characteristic regimes as a function of the scattering vector $q{ }^{[4]}$

In the low- $q$ region, the intensity is constant:

$$
I \approx K \pi^{2} R^{4} e^{2}
$$

where $K$ is a proportionality constant, $R$ is the in-plane radius of the platelet, and $\mathrm{e}$ is the platelet thickness. In the regime of intermediate scattering vectors, the intensity becomes proportional to $q^{-2}$ :

$$
I \approx \frac{K 2 \pi^{2} R^{2} e^{2}}{q^{2}}
$$

and in the wide angle regime, the intensity becomes proportional to $q^{-4}$ :

$$
I \approx \frac{K 4 \pi^{2} R^{2}}{q^{4}}
$$

The transition between these regimes occurs for a $q$-value of $\sqrt{2} / R$ and $\sqrt{2} / e$. Therefore, in the case where one has access to the full $q$-range necessary for measuring these three regimes, both the thickness and radius can be measured with good accuracy. However, depending on the aspect ratio, it can be quite challenging to obtain the necessary $q$-range. For instance, for $T_{1}$ platelets in Al alloys, the thickness of one unit cell is about $1 \mathrm{~nm},{ }^{[32,35]}$ while the diameter is usually between 50 and $100 \mathrm{~nm}$. For this case, the transitions between the above-mentioned regimes occur for $q$-values of $0.0014 \AA^{-1}$ and $0.14 \AA^{-1}$. A measurement of good quality of the full signal would then require a $q$-range between at least $5 \times 10^{-4} \AA^{-1}$ and $0.4 \AA^{-1}$, which requires a specific set-up and several sample-to-camera lengths. In the case when only a limited $q$-range is available and only one regime transition can be observed, a partial analysis (either of the thickness or the diameter of the platelets) can still be achieved.

As an example of application, Figure 2(a) shows a SAXS image recorded in the cross section of a friction stir weld of an Al-Cu-Li AA2050 alloy, which was welded in the near peak-aged condition, and therefore contained initially $T_{1}-\mathrm{Al}_{2} \mathrm{CuLi}$ platelet precipitates. The image shows some streaks that are characteristic of the presence of the platelet precipitates; however, the plastic deformation induced by the friction stir welding process results in a rotation of the texture and thus in a significant radial averaging of the signal. Figure 2(b) shows the radially averaged intensity in two areas of the friction stir weld, one in the unaffected material (base metal BM), and the other in the thermally affected zone (TMAZ) where the material has been subjected to a rapid heat spike around $623 \mathrm{~K}\left(350{ }^{\circ} \mathrm{C}\right)$. In this graph, the regimes in $q^{-2}$ and $q^{-4}$ presented above are clearly 


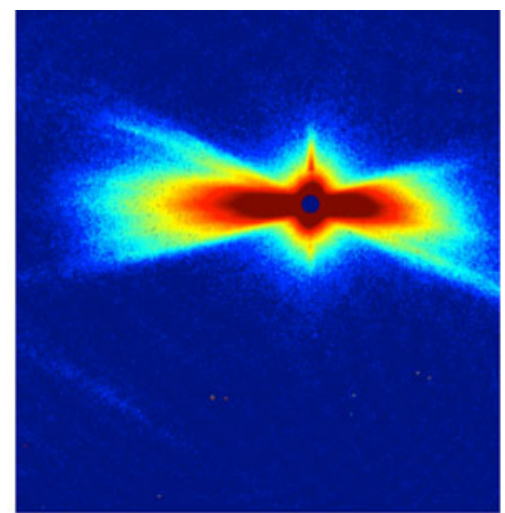

(a)

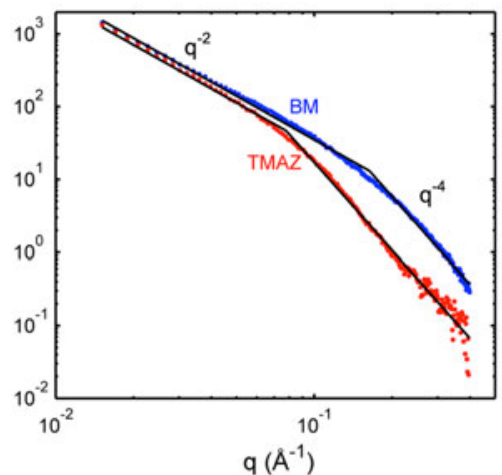

(b)

Precipitate thickness $(\AA)$

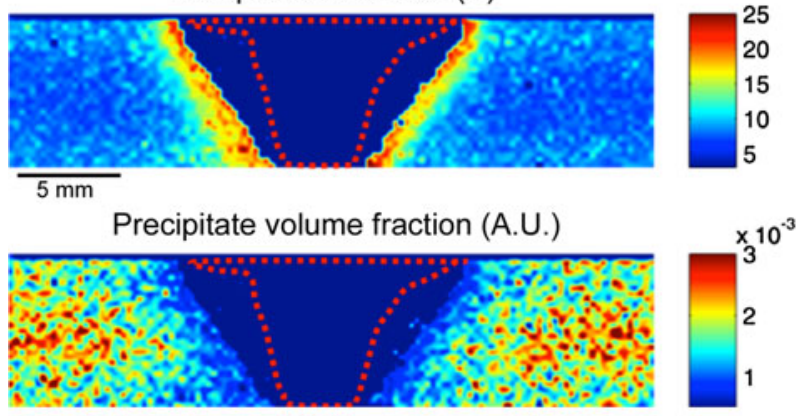

(c)

Fig. 2-(a) Example of a SAXS image recorded in the cross-section of a friction stir weld of an AA2050-T8 Al-Cu- $\mathrm{Li}$ alloy. (b) Radially averaged plots of the SAXS intensity in the base metal (BM) and thermo-mechanically affected zone (TMAZ) showing the $q^{-2}$ and $q^{-4}$ regimes, whose transition can be related to the thickness of the precipitates. $(c)$ maps of the distribution of $T_{1}$ precipitate thickness and precipitate volume fraction (in arbitrary units) in the weld cross-section (the red dashed line delineates the friction processed zone).

visible, and the transition between the two gives access to an estimate of the precipitate thickness. It appears very clearly that the heat spike in the TMAZ results not only in a partial dissolution of the precipitates, but also in an increase of the thickness of the surviving particles, which has been confirmed by TEM observations. Measuring the transition scattering vector between the $q^{-2}$ and $q^{-4}$ regimes, it was possible to automatically evaluate the thickness of the platelets on all the measurements made in the weld cross section (more than 3000 SAXS images). Note that with the measurement procedure used in the present case, the lowest scattering vector available was not sufficiently small to allow for the observation of the constant intensity regime at very small scattering vectors, so that the plate diameter could not be determined. In parallel, however, the overall intensity was measured, which gives a qualitative evaluation of the overall precipitate volume fraction. Figure 2(c) shows the corresponding maps of scattering intensity, which enable us to identify the extent of the dissolution zone and of the zone of increased precipitate thickness.

\section{Platelet Precipitates: Anisotropic Scattering, Application to the Separation of Several Precipitate Families}

In many cases, there is only a partial averaging of the scattering signal from anisotropic precipitates. The extreme case is of course the probing of a single crystal. Measuring precipitate morphologies from a single crystal of the matrix phase has been discussed in the literature. ${ }^{[3]}$ Platelets, when viewed edge-on by the beam, give rise to a scattering streak, as shown in Figure 3(a) in the case of a mixture of $\theta^{\prime}$ and $T_{1}$ precipitates present in an Al-Cu-Li alloy. Schematically, the information about the thickness $(t)$ of the platelets is inversely proportional to the extension of the streak in the reciprocal space, and the information about the diameter $(D)$ of the platelets is inversely proportional to the lateral dimension of the streak (Figure 3(a)). A simplified description of the scattering behavior of platelets in single crystals has been carried out by Fratzl et al. ${ }^{[36]}$ However, a full description requires taking into account properly the effect of precipitate size distributions and most importantly the curvature of the Ewald sphere, that modifies very significantly the scattering behavior. As shown in Figure 3(b), the anisotropic nature of the scattering signal that originates from a platelet observed edge-on results in a much faster decrease of the intensity with increasing $q$ as compared to what would be calculated without talking into account the Ewald sphere curvature (thus considering $\left.q_{y}=0\right)$.

A second complication arises when measuring precipitates in materials where the matrix presents a distribution of crystal orientations with respect to the beam. This happens for instance in highly textured polycrystals, when the texture is sufficiently strong so that the individual streaks from the precipitate variants are clearly visible, but where there are significant misorientations of the precipitates with respect to the beam. Then, two types of misorientations need to be considered that result in a very different signature on the scattering behavior:

(a) "Tilt" misorientations, namely misorientations of the habit plane of the edge-on platelet, which rotation axis is within the sample plane. In this case, the position of the streak on the detector remains strictly the same; however, the scattered intensity 


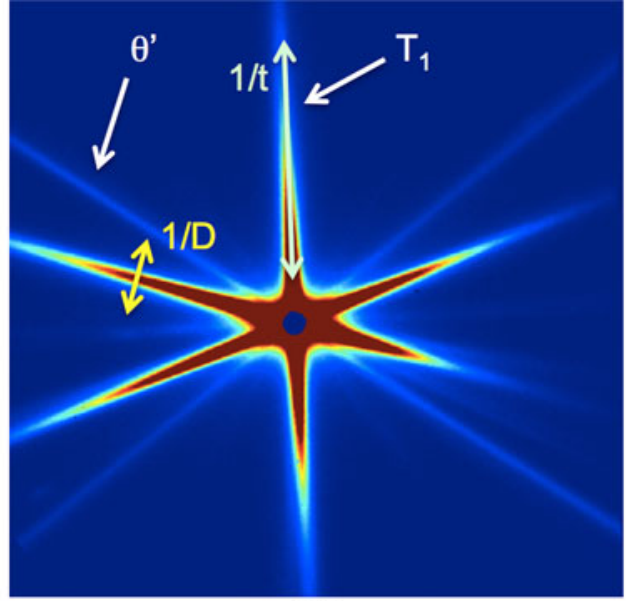

(a)

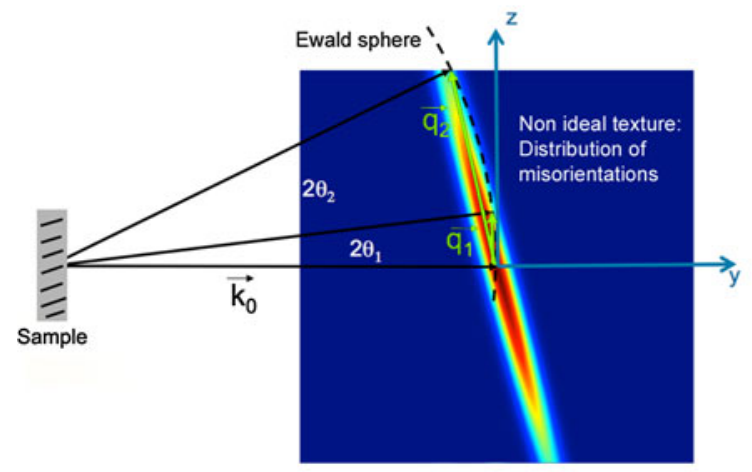

(c)

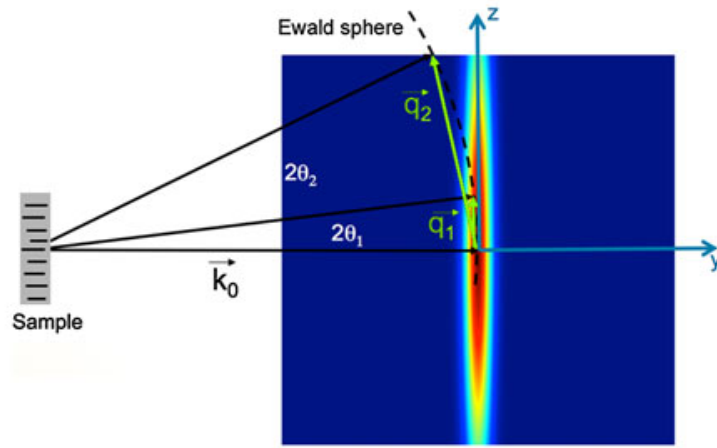

(b)

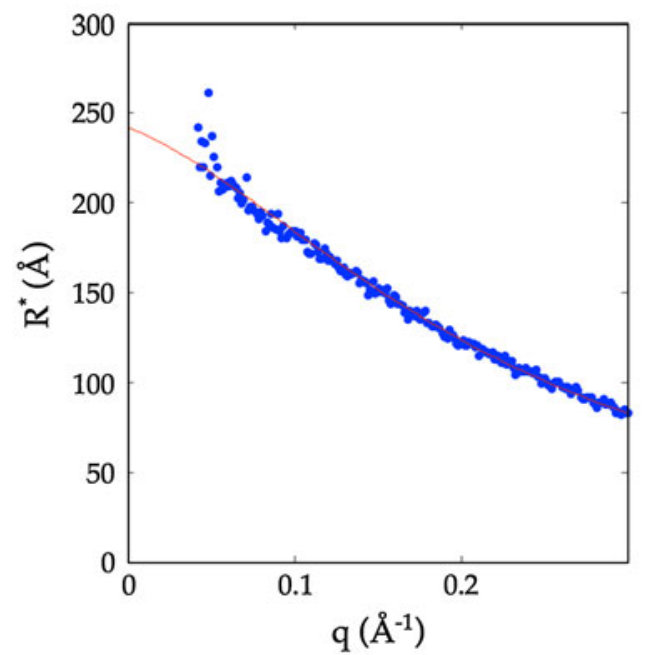

(d)

Fig. 3- (a) Example of scattering diagram recorded on a textured polycrystal of Al-Cu-Li alloy containing platelet precipitates. (b) Ewald sphere construction in the case of a scattering streak originating from a platelet precipitate illuminated edge-on by the X-ray beam, showing the necessity to perform Ewald sphere curvature corrections to describe the evolution of scattering intensity with scattering vector. (c) Similar construction when the precipitate has a tilt misorientation with respect to the X-ray beam. (d) When a twist misorientation of edge-on platelets is present, their apparent radius, which can be measured from the streak width for different scattering vectors, is not constant. The extrapolation to zero scattering vector provides the actual value of the plate radius.

varies rapidly, for a given scattering angle, as the precipitate comes in and out of the Bragg condition, as shown in Figure 3(c) (intersection of the $3 \mathrm{D}$ scattering streak with the Ewald sphere). A distribution of misorientations therefore results in a distribution of intensities along the streak. If the distribution of misorientations is relatively uniform, this effect partly cancels the corrections required to take into account the effect of the Ewald sphere curvature since for any scattering angle, there will be some precipitates that satisfy the Bragg condition and therefore give rise to the maximum possible scattering intensity.

(b) "Twist" misorientations of the precipitate habit plane, which rotation axis is normal to the sample plane. These rotations average the streak radially.
If these misorientations are sufficiently large, the individual streaks cannot be individually distinguished and the situation becomes close to that of the isotropic scattering discussed in Section I-B. However, if this misorientation is small (not more than a few degrees), then it is still possible to obtain the morphological information from the streaks by evaluating the angular dependence of the streak width (providing a measurement of the apparent plate radius), as shown in Figure 3(d). The actual plate radius corresponding to a measurement for zero misorientation is obtained by extrapolation of this behavior to a zero scattering angle. An interesting aspect about measurement resolution that was already pointed out by Fratzl et $a l^{[36]}$ is that the pixel size of the detector is 
usually much smaller than the size of the beamstop, so that the measurement of platelet diameters of very large dimensions is possible using the lateral dimensions of scattering streaks, when the measurement of such dimensions would be impossible in the case of an isotropic distribution of objects such as described in Section I-B.

It is possible to take into account the effect of both of these misorientations and obtain a faithful quantification of the precipitate morphology. For more details about this measurement procedure, refer to. ${ }^{[37]}$

One interesting application of such anisotropic measurements lies when several precipitate types occur in competition on different habit planes or with different morphologies. In this case, their contribution to the scattering intensity can be separately evaluated since they give rise to signals in different regions of the reciprocal space. In the case of $\mathrm{Al}-\mathrm{Cu}-\mathrm{Li}$ alloys, several phases are in competition for the solute: the $T_{1}$ $\mathrm{Al}_{2} \mathrm{CuLi}$ phase on the $\{111\}_{\mathrm{Al}}$ planes, the $\theta^{\prime}-\mathrm{Al}_{2} \mathrm{Cu}$ phase (or, at lower temperature, GP zones) on the $\{100\}$ Al planes, and spherical objects that can either be solute clusters or the $\theta^{\prime}-\mathrm{Al}_{3} \mathrm{Li}$ phase depending on the alloy composition. If the alloy texture allows measuring the SAXS signal with a major grain orientation along a $\langle 110\rangle$ zone axis, two $T_{1}$ variants can be observed edge-on, while one $\theta^{\prime}$ variant is in the same situation (Figure 3(a)). The spherical precipitates give rise to an isotropic scattering signal, and therefore their contribution can be obtained in between the scattering streaks of the other precipitates. In such an alloy system, the respective contribution of all these particles can therefore be obtained along any thermal path, which helps greatly to understand the complicated precipitation pathway. Figure 4(a) shows a sequence of $\mathrm{SAXS}$ images recorded on an $\mathrm{Al}-\mathrm{Cu}-\mathrm{Li}$ alloy along a heat treatment starting from a T351 temper (naturally aged after solution treatment), followed by a heating ramp to $428 \mathrm{~K}\left(155^{\circ} \mathrm{C}\right)$, and a heat treatment at $428 \mathrm{~K}$ $\left(155^{\circ} \mathrm{C}\right)$, and Figure $4(\mathrm{~b})$ shows the result of the quantitative analysis performed on the SAXS images. During the full heat treatment, the isotropic SAXS signal gives the information about the evolution of the $\delta^{\prime}$ precipitates; their size is observed to regularly increase from 2 to more than $10 \mathrm{~nm}$. After about 7 hours at $428 \mathrm{~K}\left(155^{\circ} \mathrm{C}\right)$, the apparition of streaks on the SAXS images is the signature of the nucleation of the $T_{1}$ platelets. Their diameter is observed to rapidly increase up to more than $50 \mathrm{~nm}$, while their thickness remains extremely stable at about $1 \mathrm{~nm}$, which corresponds to a platelet of one single unit cell in the thickness direction.
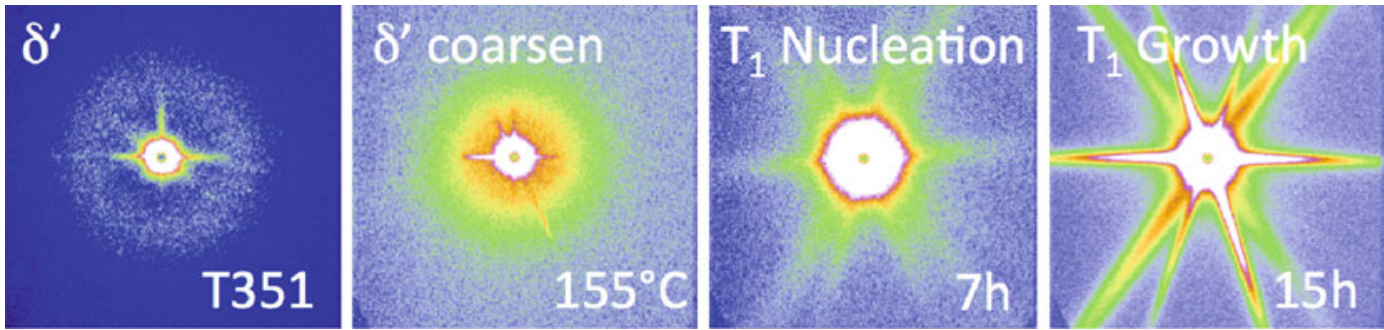

(a)

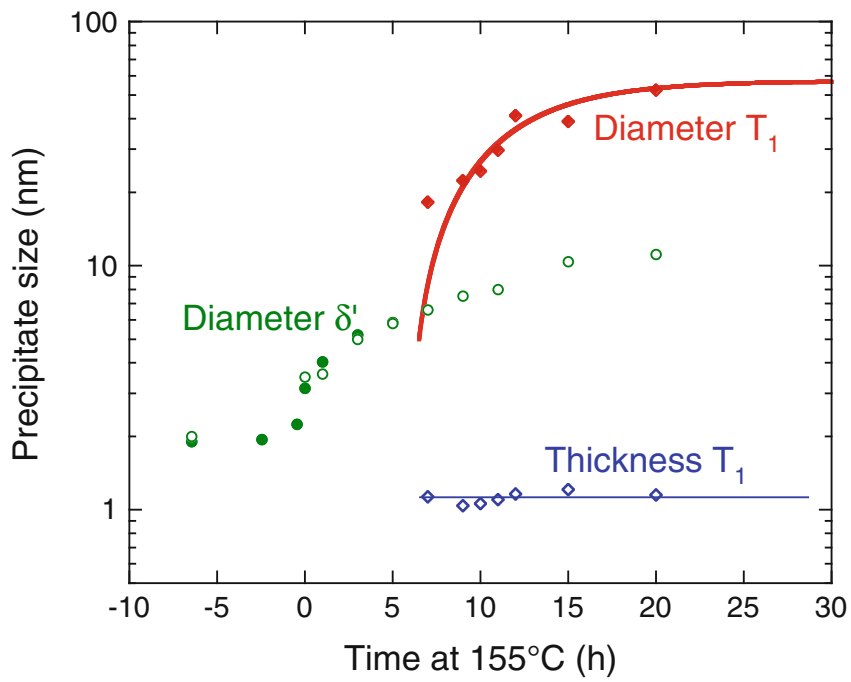

(b)

Fig. 4 - (a) Sequence of SAXS images along a heat treatment at $428 \mathrm{~K}\left(155^{\circ} \mathrm{C}\right)$ realized on an AA2196 Al-Cu-Li alloy initially in the T351 state containing $\delta^{\prime}$ precipitates. (b) Evolution of the diameter of the $\delta^{\prime}$ precipitates (full symbols are SAXS data, open symbols are TEM data) and the thickness and diameter of the $T_{1}$ precipitates extracted from the SAXS images along the heat treatment. 


\section{Considerations About Measurements of Volume Fractions and Precipitate Composition}

This paper has been devoted until now to the description of the methods that can be applied to obtain information of the precipitate size and morphology in a variety of situations. Obtaining information on their quantity (volume or molar fraction, which can be converted to number density using the information on precipitate size) is the other important objective of SAS measurements. However, this often turns out to be a rather complicated task. Without going greatly into the details, this section will list the possible strategies and challenges of a volume fraction measurement in smallangle scattering.

The measurement of the precipitate volume fraction is based on the assumption of a two-phase model for the microstructure, namely two phases each of constant composition, separated by an abrupt interface. Actually, the fact that some compositional variability exists within a phase should not change the analysis at first order since the averaging on the precipitate compositions is almost linear in many practical cases. ${ }^{[38]}$ In this twophase model, the volume fraction $f_{\mathrm{v}}$ is calculated from the so-called invariant or integrated intensity:

$$
Q_{\mathrm{o}}=\int_{0}^{\infty} I(q) q^{2} d q=2 \pi^{2}(\Delta \rho)^{2} f_{\mathrm{v}}\left(1-f_{\mathrm{v}}\right)
$$

where $\Delta \rho$ is the difference in the density of scattering factors between the precipitate and the matrix.

A first important point is that the use of the integrated intensity to measure unambiguously the volume fraction should be restricted to the case of precipitates of a moderate aspect ratio. Indeed, for very anisotropic precipitates, as described in the preceding sections, the measured scattered intensity depends critically on the orientation of the platelets with respect to the X-ray beam, and therefore there is no simple relationship between the recorded integrated intensity and the precipitate volume fraction.

\section{Intensity calibration}

The first necessity to obtain a precise measurement of volume fraction is to have access to absolute intensity measurements. This is actually far from an easy task. Usually, during a SAS measurement, the intensity of the direct beam is measured using an indirect method, like a photomultiplicator in front of a kapton window that scatters the beam, or a photodiode within the beamstop. The difficult part is to calibrate this measurement with respect both to the intensity of the beam in photons/s and with respect to the number of photons per unit of measurement in the SAS detector, which is usually a CCD camera for SAXS. The challenge for such measurements is that the incoming beam has a much larger intensity than that of the SAXS signal. Therefore, a measurement of the direct beam by a CCD camera can only be done after beam attenuation, which brings in turn new difficulties: firstly, measuring with a high precision the attenuation coefficient is difficult and secondly, when the beam is not perfectly monochromatic, the beam attenuation reinforces the proportion of hard $\mathrm{X}$-rays, which are difficult to account for.

Specific calibration samples have been devised for this purpose, the most convenient being currently used for the SAXS samples of glassy carbon. ${ }^{39-41]}$ Although they have proven to be extremely stable with time, their measurements on different instruments still show some scatter over $\pm 10 \mathrm{pct}$, and therefore such precision should be regarded as the best available in the current state-of-the-art technology. Even with such a calibration sample, one needs to be careful about other sources of uncertainty for absolute intensity calibration, like the measurement of sample transmission and the knowledge of the precise attenuation length of the measured sample, that requires the knowledge of its chemical composition and density.

\section{Available range of scattering vectors}

The precise measurement of precipitate volume fraction requires the measurement of the integrated intensity, which is the integral from 0 to infinity of $I \cdot q^{2}$ (Eq. [7] above). Since the experimental measurement is always made over a limited $q$-range, a precise measurement is possible only if proper extrapolations are made both for the low- $q$ and high- $q$ regions.

The extrapolation to the low- $q$ region is relatively easy in the cases where:

(a) There is no strong parasitic scattering contribution dominating at low scattering angles, e.g., streaks due to double Bragg scattering, residual scattering from large constituent particles or particles lying at the grain boundaries, ${ }^{[18,19]}$ or if such parasitic scattering can be efficiently subtracted from, e.g., their measurement in a microstructure free of precipitates.

(b) The smallest measured scattering angle is sufficiently low in order for the product $I \cdot q^{2}$ to decrease to a sufficiently small value so that the fraction of the integrated intensity lost in the beamstop is small.

If these conditions are fulfilled, an easy phenomenological extrapolation to $q=0$ can be performed (see e.g. ${ }^{[29]}$ ). When performing in situ measurements of the evolution of precipitate microstructures, the precipitates are observed to grow and coarsen, and therefore their scattering contribution is observed to shift to small angles. Therefore, it frequently occurs that the apparent integrated intensity diminishes because some signal is eventually lost into the beamstop (see e.g., ${ }^{[42]}$ ).

The extrapolation to the large- $q$ region depends on having a good model for the asymptotic behavior of the scattered intensity. When precipitates have a sharp interface with the matrix, the asymptotic behavior follows the so-called Porod behavior with the intensity proportional to $q^{-4}$, which can be easily integrated to infinity. ${ }^{[29]}$ Note that a constant background is added to this behavior, which can be due to not only Laue scattering, but also fluorescence when some chemical elements of the alloy have a $K$-edge slightly lower than that used for the measurements. For the case of neutron scattering, this constant intensity corresponds also to the 
contribution of incoherent scattering. The subtraction of this constant scattering is easy when the signal of the precipitates is sufficiently large, but can become problematic in low contrast situations.

Other problems can arise when measuring very small objects, such as solute clusters. ${ }^{[43]}$ First, the sharp interface model may not be valid for such objects and in any case, the measurement range is usually not sufficient so that a clear Porod behavior can be measured, and the extrapolation may therefore become somewhat subjective.

\section{Knowledge of phase composition for volume fraction} measurement

Let us assume now that we have a precisely calibrated intensity in absolute units, and that the integrated intensity can be measured with appropriate extrapolations to zero and infinity. To convert this integrated intensity into volume fraction, it is still required to know the contrast in scattering factor between the precipitates and the matrix. This is relatively straightforward when dealing with relatively large and stoichiometric precipitates. For X-rays, the scattering factor of each chemical species $i$ is simply the sum of the non-resonant scattering factors (equal to the atomic number $Z_{i}$ ) plus the resonant (anomalous) corrections, with both real (coherent, $f_{i}^{\prime}$ ) and imaginary (absorption, $f_{i}^{\prime \prime}$ ) contributions:

$$
f_{i}=Z_{i}+f_{i}^{\prime}+i f_{i}^{\prime \prime}
$$

Then, the scattering factor density in each phase (precipitate and matrix) is obtained by dividing the average scattering factor by the average atomic volume within the phase.

$$
\rho=\frac{\sum_{i} f_{i} X_{i}}{\Omega}
$$

where $X_{i}$ is the individual atomic fraction of each species $i$ and $\Omega$ is the average atomic volume. For neutrons, the scattering factors are given by the coherent scattering lengths, which depend on the distribution of isotopes for all the elements, and are well tabulated in the literature. ${ }^{[4,45]}$

When dealing with non-stoichiometric precipitates, it is obviously more difficult to obtain the scattering factor density contrast between the precipitates and the matrix. This can happen in multi-constituent alloys where solute substitutions can happen, and more generally in very small precipitates (less than $5 \mathrm{~nm}$ typically), the composition of which usually strongly departs from the equilibrium one (the presence of metastable precursors, out-of-equilibrium concentration during nucleation). In this case, it is not possible to obtain the volume fraction if more information on the precipitate composition is not obtained by another method. Among such methods, one can cite the following:

(a) Independent measurements of precipitate composition using techniques in the direct space. Analytical TEM (EELS, EDX,...) can provide useful information, although quantitative information about objects of a few nm embedded in a matrix is difficult to obtain. The most versatile method for such measurements seems to be Atom Probe Tomography, which can give valuable information on precipitate composition at the nanoscale. APT measurements for very small precipitates should also be treated with caution as they are often influenced by the matrix main elements, for instance, due to differences in evaporation field between the precipitates and matrix that cause some smearing of the atomic trajectories. However, such measurements have numerously proven very useful in conjunction to SAS measurements to obtain information on the composition and volume fraction of very small precipitates or even clusters. ${ }^{[30,38,46-54]}$

(b) In the framework of SAS measurements, contrast methods can be applied to obtain some chemical selectivity and therefore information on the composition of the precipitates. Three contrast methods can be listed that apply to precipitation in metals. The first is the use of Anomalous SAXS (ASAXS), where measurements are carried out at several wavelengths close (and slightly below, to avoid fluorescence) to an absorption edge of the element of interest. ${ }^{[55]}$ Although ASAXS provides valuable information on the presence of a chemical element in the particles under study, it is not usually sufficient for an independent measurement of the composition of this element, and hypothesis or

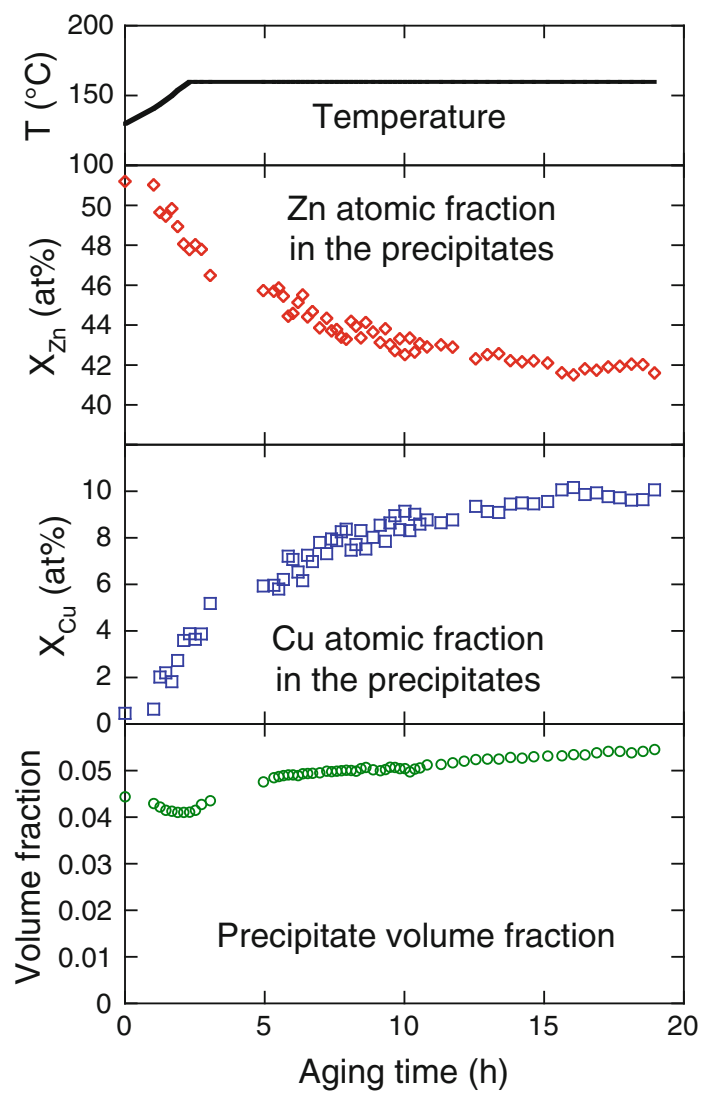

Fig. 5-Measurement by in-situ ASAXS of the evolution of volume fraction and precipitate composition in an $\mathrm{Al}-\mathrm{Zn}-\mathrm{Mg}-\mathrm{Cu}$ alloy during an aging treatment at $433 \mathrm{~K}\left(160^{\circ} \mathrm{C}\right)$. 
independent measures are generally required. ${ }^{[56]}$ The second method is specific to the study by SANS of magnetic materials containing nonmagnetic precipitates, which is the general case for steels. In this case, SANS studies are generally performed under a saturating magnetic field, giving access to both the magnetic and nuclear SAS spectra. The ratio between these two signals depends on the compositions of the matrix and precipitates and can therefore be compared to a different hypothesis made on the precipitate composition. ${ }^{[9,11,14,50,57]}$ Finally, the SANS and SAXS signals can also be compared in a similar way, giving additional information on the composition of particles. ${ }^{[15]}$

As an example of application of such methods, Figure 5 shows the measurement of precipitate volume fraction and composition of $\mathrm{MgZn}_{2}$-type precipitates in an $\mathrm{Al}-\mathrm{Zn}-\mathrm{Mg}-\mathrm{Cu}$ alloy (see ${ }^{[56]}$ for more details). Using Atom Probe Tomography, it was possible to assess that the composition in $\mathrm{Mg}$ and $\mathrm{Al}$ during the heat treatment was relatively constant. ASAXS measurements carried out in situ during the heat treatment at the $\mathrm{Zn}$ and $\mathrm{Cu}$ edges provided the evolution of the precipitate composition in these two species. It was then possible to access the volume fraction, and to show that a partial substitution of $\mathrm{Zn}$ by $\mathrm{Cu}$ occurred progressively during the heat treatment.

\section{CONCLUSIONS}

Characterizing quantitatively the parameters of a precipitate microstructure in a metallic system by smallangle scattering brings some specific difficulties that include the simultaneous presence of a size distribution of the objects, complex morphologies, and a nonrandom texture that causes some, and yet imperfect, averaging of the signal. We have seen in this paper that several strategies can be devised to analyze the data, depending on some limiting cases: spherical or nearspherical particles, particles of high aspect ratio, close to random texture or very strong texture. If this analysis is carefully carried out, a robust and fast result can be obtained, which opens the way to automated analysis of large datasets such as those obtained with synchrotron data during in situ measurements or mapping of heterogeneous microstructures.

\section{ACKNOWLEDGMENTS}

The technical staff of beamline BM02-D2AM of ESRF is gratefully acknowledged for technical support. Dr. F. Bley is thanked for fruitful discussions. The French research agency (ANR) is thanked for financial support under the projects ALICANTDE and CORALIS (ANR-08-MAPR-0020-05). Dr. J.C. Ehrström, C. Sigli, and C. Hénon from ConstelliumCRV, and J. Delfosse from EADS-IW are thanked for their continued support and discussions during these projects. A.D. wishes to acknowledge the support of the European Research Council for support in the framework of the NEMOLight Marie Curie International Outgoing Fellowship within the 7th European Community Framework Programme.

\section{REFERENCES}

1. A. Guinier: Nature, 1938, vol. 142, p. 569.

2. A. Guinier and G. Fournet: Small-Angle Scattering of X-rays, Wiley, New York, 1955.

3. V. Gerold and G. Kostorz: J. Appl. Cryst., 1978, vol. 11, pp. 376404.

4. O. Glatter and O. Kratky: Small Angle X-Ray Scattering, Academic Press, London, 1982.

5. P. Fratzl: J. Appl. Cryst., 2003, vol. 36, pp. 397-404.

6. P. Staron, B. Jamnig, H. Leitner, R. Ebner, and H. Clemens: $J$. Appl. Cryst., 2003, vol. 36, pp. 415-19.

7. D. Del Genovese, P. Strunz, D. Mukherji, R. Gilles, and J. Rosler: Metall. Mater. Trans. A, 2005, vol. 36A, p. 3439-3450.

8. F. Perrard, A. Deschamps, F. Bley, P. Donnadieu, and P. Maugis: J. Appl. Crystallogr., 2006, vol. 39, pp. 473-82.

9. M.J. Alinger, G.R. Odette, and D.T. Hoelzer: Acta Mater., 2009, vol. 57 , pp. 392-406.

10. T. Marlaud, A. Deschamps, F. Bley, W. Lefebvre, and B. Baroux: Acta Mater., 2010, vol. 58, pp. 4814-26.

11. P. Michaud, D. Delagnes, P. Lamesle, M. Mathon, and C. Levaillant: Acta Mater., 2007, vol. 55, pp. 4877-89.

12. M. Kusy, P. Riello, and L. Battezzati: Acta Mater., 2004, vol. 52, pp. $5031-41$

13. M. Bischof, P. Staron, D. Caliskanoglu, H. Leitner, C. Scheu, and H. Clemens: Mater. Sci. Eng. A, 2008, vol. 472, pp. 148-56.

14. H. Leitner, P. Staron, H. Clemens, S. Marsoner, and P. Warbichler: Mater. Sci. Eng. A, 2005, vol. 398, pp. 323-31.

15. M. Ohnuma, J. Suzuki, S. Ohtsuka, S.-W. Kim, T. Kaito, M. Inoue, and H. Kitazawa: Acta Mater., 2009, vol. 57, pp. 5571-81.

16. O. Glatter: J. Appl. Cryst., 1977, vol. 10, pp. 415-21.

17. O. Glatter: J. Appl. Cryst., 1980, vol. 13, pp. 7-11.

18. M. Kompatscher, B. Deme, G. Kostorz, C. Somsen, and E.F. Wassermann: Acta Mater., 2002, vol. 50, pp. 1581-86.

19. D. Mukherji, P. Strunz, D.D.E.L. Genovese, R. Gilles, J. Rosler, and A. Wiedenmann: Metall. Mater. Trans. A, 2003, vol. 34A, pp. 2781-92.

20. D.J. Kinning and E.L. Thomas: Macromolecules, 1984, vol. 17, p. 1712.

21. B. Weyerich, J. Brunner-Popela, and O. Glatter: J. Appl. Crystallogr., 1999, vol. 32, p. 197.

22. A. Deschamps, M. Militzer, and W.J. Poole: ISIJ Int., 2001, vol. 41 (2), pp. 196-205.

23. T. Kamiyama, H. Kimura, K. Sasamori, and A. Inoue: Scripta Mater., 2001, vol. 44, pp. 1297-1301.

24. M. Ohnuma, K. Hono, S. Linderoth, J.S. Pedersen, Y. Yoshizawa, and H. Onodera: Acta Mater., 2000, vol. 48, pp. 4783-90.

25. A. Dupasquier, R. Ferragut, M.M. Iglesias, M. Massazza, G. Riontino, P. Mengucci, G. Barucca, C.E. Macchi, and A. Somoza: Phil. Mag., 2007, vol. 87, pp. 3297-23.

26. P. Kenesei, G. Horvath, S. Bernstorff, T. Ungar, and J. Lendvai: Zeit. Metallk., 2006, vol. 97, pp. 315-20.

27. J.B. Wiskel, D.G. Ivey, and H. Henein: Metall. Mater. Trans B, 2008, vol. 39B, pp. 116-24

28. A. Deschamps and F. De Geuser: J. Appl. Cryst., 2001, vol. 44, pp. $343-52$.

29. F. De Geuser and A. Deschamps: C. R. Physique, 2012, vol. 13, pp. 246-56.

30. A. Deschamps, C. Sigli, T. Mourey, F. de Geuser, W. Lefebvre, and B. Davo: Acta Mater., 2012, vol. 60, pp. 1917-28.

31. S.K. Son, M. Takeda, M. Mitome, Y. Bando, and T. Endo: Mater. Lett., 2005, vol. 59, pp. 629-32.

32. P. Donnadieu, Y. Shao, F. De Geuser, G.A. Botton, S. Lazar, M. Cheynet, M. de Boissieu, and A. Deschamps: Acta Mater., 2011, vol. 59 , pp. $462-72$ 
33. J.F. Nie, X.L. Xiao, C.P. Luo, and B.C. Muddle: Micron, 2001, vol. 32 , pp. 857-63.

34. J.C. Oh, T. Ohkubo, T. Mukai, and K. Hono: Scripta Mater., 2005, vol. 53, pp. 675-79.

35. C. Dwyer, M. Weyland, L.Y. Chang, and B.C. Muddle: Appl. Phys. Lett., 2011, vol. 98, p. 201909.

36. P. Fratzl, F. Langmayr, and O. Paris: J. Appl. Cryst., 1993, vol. 26, pp. $820-26$.

37. F De Geuser and A Deschamps: J. Appl. Crystallogr., 2011, vol. 44 , p. 2.

38. M. Dumont, W. Lefebvre, B. Doisneau-Cottignies, and A. Deschamps: Acta Mater., 2005, vol. 53, pp. 2881-92.

39. F. Zhang, J. Ilavsky, G.G. Long, J.P.G. Quintana, A.J. Allen, and P.R. Jemian: Metall. Mater. Trans. A, 2010, vol. 41A, pp. 1151-58.

40. L. Fan, M. Degen, S. Bendle, N. Grupido, and J. Ilavsky: J. Phys. Conf. Ser., 2010, vol. 247, p. 012005.

41. C.A. Dreiss, K.S. Jack, and A.P. Parker: J. Appl. Cryst., 2006, vol. 39, pp. 32-38.

42. B. Wattiez, A.F. Gourgues, A. Deschamps, A. Roemer, and Z. Zermout: Mater. Sci. Eng. A, 2010, vol. 527, pp. 7901-11.

43. A. Deschamps, T.J. Bastow, F. De Geuser, A.J. Hill, and C.R. Hutchinson: Acta Mater., 2011, vol. 59, pp. 2918-27.

44. G.E. Bacon: Neutron Diffraction, Oxford University Press, UK, 1962.

45. http://www.ncnr.nist.gov/resources/n-lengths/.

46. A. Deschamps, A. Bigot, F. Livet, P. Auger, Y. Brechet, and D. Blavette: Phil. Mag. A, 2001, vol. 81, pp. 2391-2414.
47. W. Lefebvre, K. Hoummada, B. Decreus, F. de Geuser, and A. Deschamps: Proceedings of the 11th International Conference on Aluminium Alloys, J. Hirsch, B. Skrotzki, and G. Gottstein, eds., Wiley, Germany, 2008, pp. 801-07.

48. M. Bischof, S. Erlach, P. Staron, H. Leitner, C. Scheu, and H. Clemens: Zeit. Metallk., 2005, vol. 96, pp. 1074-80.

49. H. Leitner, H. Clemens, J. Akre, F. Danoix, and P. Staron: Int. J. Mater. Res., 2007, vol. 98, pp. 1093-1103.

50. E. Eidenberger, M. Schober, E. Stergar, H. Leitner, P. Staron, and H. Clemens: Metall. Mater. Trans. A, 2010, vol. 41A, pp. 1230-34.

51. M. Schober, C. Lerchbacher, E. Eidenberger, P. Staron, H. Clemens, and H. Leitner: Intermetallics, 2010, vol. 18, pp. 155359.

52. Z.W. Zhang, C.T. Liu, X.-L. Wang, K.C. Littrell, M.K. Miller, K. An, and B.A. Chin: Phys. Rev. B, 2011, vol. 84.

53. M.K. Miller, B.D. Wirth, and G.R. Odette: Mater. Sci. Eng. A, 2003, vol. 353, pp. 133-39.

54. Z.K. Teng, C.T. Liu, M.K. Miller, G. Ghosh, E.A. Kenik, S. Huang, and P.K. Liaw: Mater. Sci. Eng. A, 2012, vol. 541, pp. 22-27.

55. G. Goerigk, H.-G. Haubold, O. Lyon, and J.P. Simon: J. Appl. Cryst., 2003, vol. 36, pp. 425-29.

56. T. Marlaud, A. Deschamps, F. Bley, W. Lefebvre, and B. Baroux: Acta Mater., 2010, vol. 58, pp. 248-60.

57. M. Bischof, P. Staron, A. Michels, P. Granitzer, K. Rumpf, H. Leitner, C. Scheu, and H. Clemens: Acta Mater., 2007, vol. 55, pp. 2637-46. 